\title{
DOMINATED STRATEGIES IN SEARCHING FOR EVOLUTIONARY STABLE STRATEGIES
}

\author{
P. BREEN ${ }^{1}$ AND $W$. HENDERSON ${ }^{2}$
}

(Received 15 August 1986; revised 1 February 1988)

\begin{abstract}
The paper asks and answers the question "When does dominance of a particular strategy play a role in the search for evolutionary stable strategies?" The answer is much less obvious than would appear at first glance.

When there is strict dominance of a pure strategy, it is clear that the dominated strategy should never be employed in any conflict. However, when the dominance is not strict it is less obvious that the strategy should not be used. The research was originally intended to clear up this grey area in the theory of evolutionary stable strategies, but it has turned out to be of more than simply academic interest. The result can be used, with varying degrees of success, to simplify the search procedure for these evolutionary stable strategies when a reward matrix is given.
\end{abstract}

\section{Introduction}

Evolutionary stable strategies (ESS's) were introduced by Maynard Smith and Price [6] to model the evolution of animal behaviour in conflict situations. A strategy is a probability distribution $q$ on the $n$ pure strategies which the animals can use in the conflict. A strategy can therefore be thought of as the random way a single animal uses various pure strategies or, more accurately, the way in which the population as a whole mixes up the available pure strategies. An ESS is a strategy which has sufficient strength and flexibility when measured in mean fitness terms to outlast any other possible strategy. We define an ESS mathematically in Section 2.

\footnotetext{
${ }^{1}$ Slab and Plate Product Division, B. H. P. Steel, Pt. Kembla, N. S. W. 2505, Australia.

${ }^{2}$ Applied Mathematics Department, University of Adelaide, G.P.O. Box 498, Adelaide, S.A. 5001, Australia.
}

(C) Copyright Australian Mathematical Society 1988, Serial-fee code 0334-2700/88 
The idea of Maynard Smith and Price[6] was to treat the conflict as a game and parallel some of the ideas of game theory in order to develop results on ESS's. Later authors, such as Haigh [4], Bishop and Cannings [3] and Abakuks [1], developed a search procedure to find all possible ESS's whenever the reward matrix is given. For an excellent survey of the theory and problems of ESS's see Maynard Smith [5].

This paper looks at the game theory idea of dominance applied to ESS's. In particular, the relationship between weak dominance of a pure strategy by another strategy and the use of that pure strategy is an ESS. The theorem, given in Section 3, not only provides more information on the structure of ESS's but may also help to simplify the search procedure by the elimination from consideration of all submatrices with obviously dominated rows and the reduction in size from $n$ to $n-1$ of the original matrix when one of its rows is weakly dominated. Considering that the search procedure involves studying up to $2^{n}-1$ square matrices, every little helps.

Akin [2] also discusses dominance in ESS's using an approach attributable to Taylor and Jonker [7]. Akin, however, considers only "interior" strategies, that is, strategies which use every pure strategy with positive probability. This paper does not exclude interior strategies, but is generally more concerned with ESS's which lie on the boundary of the space of potential strategies.

Although the proofs given here are for the finite strategy-space case, the extensions to countably infinite and continuous strategy spaces are straightforward.

\section{Definitions and basic results}

Let $\Lambda=\left\{\mathbf{p} \in R^{n}: p_{i} \geq 0 \forall 1 \leq i \leq n, \sum_{i=1}^{n} p_{i}=1\right\}$ be the set of all strategies. Let $\mathbf{p}, \mathbf{q} \in \Lambda$ and $\mathbf{A}=\left\{a_{i j}: 1 \leq i, j \leq n\right\}$ be an $n \times n$ reward matrix. Define $R(\mathbf{p})=\left\{k: p_{k}>0\right\}$ to be the support of strategy $\mathbf{p}, E(k, \mathbf{p})=$ $\sum_{j=1}^{n} a_{k j} p_{j}=\left\{A \mathbf{p}^{T}\right\}_{k}$ is the payoff using pure strategy $k$ against strategy $\mathbf{p}$ and $E(\mathbf{q}, \mathbf{p})=\mathbf{q} A \mathbf{p}^{T}$ is the payoff using strategy $\mathbf{q}$ against strategy $\mathbf{p}$. Define $\mathbf{p}$ to be an ESS if and only if

$$
E(\mathbf{p}, \mathbf{p}) \geq E(\mathbf{q}, \mathbf{p}) \quad \forall \mathbf{q} \in \Lambda
$$

and

$$
E(\mathbf{p}, \mathbf{q})>E(\mathbf{q}, \mathbf{q}) \quad \text { whenever } E(\mathbf{p}, \mathbf{p})=E(\mathbf{q}, \mathbf{p}) .
$$

Equation (1) tells us that the reward using strategy $\mathbf{p}$, in a population playing p, must be at least as good as using any other strategy. Equation (2) considers the situation in which a "rebel" strategy $q$ gains the same rewards as $p$ in the population playing $\mathbf{p}$. If the rebels build up in strength, then strategy $\mathbf{p}$ 
reasserts itself by gaining more than $\mathbf{q}$ when in conflict with the rebels. Bishop and Cannings [3] have shown that if $\mathbf{p}$ is an ESS then

$$
\begin{aligned}
E(k, \mathbf{p}) & =E(\mathbf{p}, \mathbf{p}) \quad \forall k \in R(\mathbf{p}) \\
& =\max _{1 \leq j \leq n} E(j, \mathbf{p}) .
\end{aligned}
$$

Also let $S(\mathbf{p})=\{k: E(k, \mathbf{p})=E(\mathbf{p}, \mathbf{p})\}$.

The strategy $\mathbf{s}$, with $s_{i}=0$, weakly dominates the pure strategy $i$ under $\mathbf{p}$ if

$$
a_{i j} \leq \sum_{k} s_{k} a_{k j}, \quad \forall j \in R(\mathbf{p}) .
$$

It is certainly true that whenever a strategy is strictly dominated, that is, when

$$
\exists \mathrm{s} \in \Lambda \text { with } s_{i}=0 \text { such that } a_{i j}<\sum_{k} s_{k} a_{k j} \forall j,
$$

the $i$ th strategy can play no part in an ESS. However, as the following theorem and the subsequent example shows, some pure strategies, and potential ESS's, may be eliminated from consideration under much weaker conditions than (6).

\section{New results}

THEOREM 1. If $A$ has an ESS $\mathbf{p}$ with $p_{i}>0$, and pure strategy $i$ is weakly dominated by $s$ under $\mathbf{p}$, then

$$
\begin{gathered}
a_{i j}=\sum_{k=1}^{n} s_{k} a_{k j} \quad \forall j \in R(\mathbf{p}), \\
R(\mathbf{s}) \subseteq S(\mathbf{p}) \\
E(i, \mathbf{s})>E(\mathbf{s}, \mathbf{s}) .
\end{gathered}
$$

The theorem indicates that, when the $i$ th pure strategy is dominated, $p_{i}$ may still be non-zero, but only if this pure strategy can maintain itself as well as the dominating a strategy against each pure strategy used by the ESS population, and then reassert itself when used against the $s$ strategy.

ProOF. Equations (3), (4) and (5) give

$$
E(\mathbf{p}, \mathbf{p})=\sum_{j \in R(\mathbf{p})} a_{i j} p_{j} \leq \sum_{k} s_{k} \sum_{j \in R(\mathbf{p})} a_{k j} p_{j} \leq \sum_{k} s_{k} \mathbf{p} A \mathbf{p}^{T}=E(\mathbf{p}, \mathbf{p}) .
$$

Equality holds throughout if and only if (7) and (8) are valid.

To show (9), consider a strategy q where

$$
\begin{aligned}
& q_{i}=0 \\
& q_{r}=p_{r}+s_{r} p_{i}, \quad \forall r \neq i .
\end{aligned}
$$


Note that $R(\mathbf{q})=R(\mathbf{p}) \cup R(\mathbf{s}) \backslash\{i\}$ and hence $E(j, \mathbf{p})=E(\mathbf{p}, \mathbf{p}) \quad \forall j \in R(\mathbf{q})$ from (3) and (8). Consequently, $E(\mathbf{q}, \mathbf{p})=E(\mathbf{p}, \mathbf{p})$.

Now if $\mathbf{p}$ is to be an ESS, (2) must hold, that is, $E(\mathbf{p}, \mathbf{q})>E(\mathbf{q}, \mathbf{q})$.

Consider

$$
\begin{aligned}
E(\mathbf{p}, \mathbf{q})-E(\mathbf{q}, \mathbf{q}) & =(\mathbf{p}-\mathbf{q}) A \mathbf{q}^{T} \\
& =p_{i}\left(A \mathbf{q}^{T}\right)_{\imath}+\sum_{k \in R(\mathbf{q})}\left(p_{k}-q_{k}\right)\left(A \mathbf{q}^{T}\right)_{k} \\
& =p_{\imath}\left(A \mathbf{q}^{T}\right)_{i}+\sum_{k \in R(\mathbf{s})}\left(-p_{\imath} s_{k}\right)\left(A \mathbf{q}^{T}\right)_{k} \\
& =p_{i}\left(\sum_{j \in R(\mathbf{q})}\left[a_{i j}-\sum_{k \in R(\mathbf{s})} s_{k} a_{k j}\right] q_{j}\right) .
\end{aligned}
$$

But if $j \in R(\mathbf{p}),(7)$ makes the bracket zero, and if $j \in R(\mathbf{q}) \backslash R(\mathbf{p}), q_{j}=s_{j} p_{i}$.

Therefore

$$
\begin{aligned}
E(\mathbf{p}, \mathbf{q})-E(\mathbf{q}, \mathbf{q}) & =p_{i}^{2}\left[\sum_{j \in R(\mathbf{s}) \backslash R(\mathbf{p})}\left(a_{i j}-\sum_{k \in R(\mathbf{s})} s_{k} a_{k j}\right) s_{j}\right] \\
& =p_{\imath}^{2}\left[\sum_{j \in R(\mathbf{s})}\left(a_{i j}-\sum_{k \in R(\mathbf{s})} s_{k} a_{k j}\right) s_{j}\right] \quad \text { from (7) } \\
& =p_{i}^{2}[E(i, \mathbf{s})-E(\mathbf{s}, \mathbf{s})] \\
& >0 \quad \text { iff } E(i, \mathbf{s})>E(\mathbf{s}, \mathbf{s}) \text { whenever } p_{\imath}>0 .
\end{aligned}
$$

COROLLARY 1. If $A$ has the ith row entirely dominated by a convex combination of the other rows, that is, $a_{i j} \leq \sum_{k} s_{k} a_{k j} \forall j$, then $p_{i}=0$ in any ESS.

ProOF. Since $a_{\imath j} \leq \sum_{k} s_{k} a_{k j} \forall j$, then

$$
\sum_{j} a_{i j} s_{j} \leq \sum_{j} \sum_{k} s_{k} a_{k j} s_{j}
$$

that is, $E(i, \mathbf{s}) \leq E(\mathbf{s}, \mathbf{8})$. This contradicts (9) and therefore $p_{i}=0$ in any ESS.

COROLLARY 2. $R(\mathbf{s}) \nsubseteq R(\mathbf{p})$ whenever the conditions of the theorem hold.

Proof. Assume $R(\mathbf{s}) \subset R(\mathbf{p})$. Then $(7) \Rightarrow E(i, \mathbf{s})=E(\mathbf{s}, \mathbf{s})$, which contradicts (9). 


\section{An example}

The theorem, as stated, is worth noting for academic completeness. As far as using it to find ESS's is concerned, the contrapositive of the theorem is of most use. That is, if (5) holds, one of (7), (8) and (9) is not satisfied, and then either $\mathbf{p}$ is not an ESS or $p_{i}=0$.

As an example, consider the reward matrix

$$
A=\left[\begin{array}{lll}
4 & 2 & 6 \\
3 & 2 & 7 \\
5 & 2 & 5
\end{array}\right]
$$

Since Row $1 \leq \frac{1}{2}$ (Row $2+$ Row 3 ), Corollary 1 is applicable and $p_{1}=0$ in any ESS.

This leaves potential ESS's of the form (i) $\mathbf{p}=(0, p, 1-p), \quad 0<p<1$, (ii) $\mathbf{p}=(0,0,1)$ and (iii) $\mathbf{p}=(0,1,0)$. For (i), $R(\mathbf{p})=\{2,3\}$. Let $\mathbf{s}=(0,1,0)$ and $i=3$. Equation (5) is then satisfied in the columns $j \in R(\mathbf{p})$. However (7) is not valid and therefore either $p$ is not an ESS or $1-p=0$. Since we assumed that $0<p<1$ a strategy $\mathrm{p}$ of the form $(0, p, 1-p)$ cannot give an ESS. For (ii), $E(\mathbf{p}, \mathbf{p})=5$ and $E(\mathbf{q}, \mathbf{p})>5$ for any $\mathbf{q} \neq \mathbf{p}$. Hence $\mathbf{p}=(0,0,1)$ is not an ESS. This leaves (iii) with $R(\mathbf{p})=\{2\}$. Let $\mathbf{s}=(1,0,0)$ and $i=2$. Equations (5), (7) and (8) are all trivially satisfied, but $E(i, s)=E(2,1)=3$ and $E(\mathbf{s}, \mathbf{s})=E(1,1)=4$. Therefore (9) is invalidated and hence $\mathbf{p}=(0,1,0)$ is not an ESS.

Consequently $\boldsymbol{A}$ has no evolutionary stable strategies.

\section{Conclusion}

In the abstract we use the statement that the result can be used, with varying degrees of success, to help find ESS's. In the introduction we use the phrases "every little helps" and "obviously dominated". Such wording is meant to convey the message that our theorem will not necessarily help find ESS's. In many situations it may be more difficult to establish a dominating vector 8 than to proceed in an alternative manner. However, without this theorem, it was never clear whether a pure strategy was dominated once a few equalities crept into the lineup, nor was it clear which columns were the most relevant in the discussion of dominance of a particular pure strategy. Both these points have now been clarified.

It can be argued that if $a_{i j}=\sum_{k} s_{k} a_{k j}$ holds for some $j \in R(\mathbf{p})$, then any ESS in which this equality plays an important role is not robust to arbitrary small perturbations in the reward matrix, and is therefore of no biological interest. 
We believe that an unstable ESS of this form would be of biological interest, especially if it were the only ESS for the conflict. There may be a tendency for the population's strategy to drift towards this point without it necessarily being a fixed point.

On the other hand, if we use the theorem to reject the $i$ th pure strategy because of a dominating strategy in which the occasional equality holds, then $i$ cannot be part of an ESS when the reward matrix can only be given to within an error factor. That is, if a strategy $i$ is dominated with some equality, it certainly cannot be trusted to be part of a stable ESS under uncertain reward conditions. Consequently the use of the theorem in rejecting strategies for inclusion in potential ESS's is still valid when small perturbations are allowed in the reward matrix. It would be of interest to see some results on the stability of ESS's in such situations.

\section{Acknowledgement}

We thank referees for bringing to our attention some of the points mentioned in the conclusion.

\section{References}

[1] A. Abakuks, "Conditions for evolutionary stable strategies", J. Appl. Prob. 17 (1980) $559-562$.

[2] E. A kin, "Domination or equilibrium", Math. Biosci. 50 (1980) 239-250.

[3] D. T. Bishop and C. Cannings, "Models of animal conflict", Adv. Appl. Prob. 8 (1976) 616-621.

[4] J. Haigh, "Game theory and evolution", Adv. Appl. Prob. 7 (1975) 8-11.

[5] J. Maynard Smith, Evolution and the theory of games, Cambridge University Press, (1982).

[6] J. Maynard Smith and G. R. Price, "The logic of animal conflict", Nature 246 (1973) 15.

[7] P. D. Taylor and L. B. Jonker "Evolutionary stable strategies and game dynamics", Math. Biosci. 40 (1978) 145-156. 